\title{
A felicidade e o engagement no trabalho nos cuidados de saúde primários
}

Sara Cunha, ${ }^{1}$ Céline Gama, ${ }^{1}$ Marta Fevereiro, ${ }^{2}$ Adriana Vasconcelos, ${ }^{2}$ Sophie Sousa, ${ }^{1}$ Ana Cristina Neves, ${ }^{2}$ Joana Casanova, ${ }^{3}$ Maria José Teixeira, ${ }^{4}$ Sandra Rodrigues, ${ }^{5}$ Susana Ribeira, ${ }^{3}$ J. Firmino-Machado ${ }^{6}$

\section{RESUMO}

Objetivos: Testar a associação entre o bem-estar psicológico (BEP) e o engagement no trabalho dos profissionais de saúde. Tipo de estudo: Estudo transversal analítico.

Local: Agrupamentos de Centros de Saúde de Matosinhos e Grande Porto IV.

População: Profissionais de saúde dos dois Agrupamentos de Centros de Saúde.

Métodos: Foram utilizadas escalas validadas para a língua portuguesa - Oxford Happiness Questionnaire (OHQ) e Utrecht Work Engagement Scale (UWES), que avaliam o BEP e o engagement no trabalho, respetivamente. Foi testada a associação entre o BEP e o engagement, controlando para variáveis de confundimento, através de um modelo de regressão linear múltipla.

Resultados: Entre os profissionais, 52,5\% são felizes ou bastante felizes e 45,5\% apresentam um alto nível de engagement. Existe uma correlação significativa entre as escalas $(r=0,32, p<0,05)$. Esta associação verifica-se mesmo quando controlada para variáveis de confundimento $(\beta=0,621, \mathrm{IC} 95 \%[0,423-0,818])$. A idade e os anos de trabalho correlacionam-se com o UWES $(r=0,16, p=0,008$ e $r=0,16, p=0,010)$ e com o OHQ $(r=-0,14, p=0,02$ e $r=-0,13, p=0,03)$.

Conclusões: $A$ associação encontrada entre BEP e engagement vai de encontro ao debatido na literatura. Maior idade e maior tempo de serviço associam-se a maior engagement, como verificado noutros estudos, mas a menor BEP. Este trabalho aborda uma temática fundamental no contexto organizativo dos cuidados de saúde primários, incentivando a implementação de estratégias que promovam o desenvolvimento pessoal e das equipas.

Palavras-chave: Trabalho; Felicidade; Cuidados de saúde primários; Satisfação no trabalho; Saúde do trabalhador.

\section{INTRODUÇÃO}

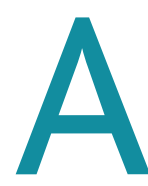

s organizações têm um papel central na vida das pessoas. O trabalho, per se, não faz uma pessoa feliz, mas uma pessoa não pode ser genuinamente feliz se for infeliz no trabalho. ${ }^{1}$

As USF (Unidades de Saúde Familiar) e as UCSP (Unidades de Cuidados de Saúde Personalizados) são unidades funcionais e multiprofissionais com aspetos similares no que respeita à sua composição, mas com diferenças na sua organização e formação. As USF distinguem-se por serem unidades funcionais com autonomia administrativa, técnica e funcional, que se

1. Médica Interna de Medicina Geral e Familiar. USF Oceanos, ACeS Matosinhos. 2. Médica Interna de Medicina Geral e Familiar. USF do Mar, ACeS Grande Porto IV. 3. Médica Assistente de Medicina Geral e Familiar. USF Oceanos, ACeS Matosinhos. 4. Secretária Clínica. USF Oceanos, ACeS Matosinhos.

5. Médica Assistente de Medicina Geral e Familiar. USF do Mar, ACeS Grande Porto IV. 6. Médico Interno de Saúde Pública. ACeS Porto Ocidental. organizam de forma voluntária. ${ }^{2}$ Assim, as USF e as UCSP regem-se por um conjunto de princípios que são compatíveis com as dimensões de uma organização positiva ou saudável, onde é possível conciliar dois objetivos transversais: desempenho e bem-estar pessoal. ${ }^{3}$ Felicidade e bem-estar psicológico (BEP) são termos que se misturam frequentemente na literatura científica e que geralmente são considerados sinónimos, ${ }^{4}$ sendo que o último conceito engloba três subdomínios: satisfação com a vida, presença de experiências emocionais positivas e ausência de experiências emocionais negativas. ${ }^{5} \mathrm{O}$ BEP pode ser avaliado através do Oxford Happiness Questionnaire (OHQ), validado para a língua portuguesa. ${ }^{6}$

O engagement no trabalho é um estado afetivo-motivacional, positivo e recompensador, relacionado com o bem-estar no trabalho. ${ }^{7}$ É composto por três subdo- 
mínios: vigor, dedicação e absorção. O vigor caracteriza-se por altos níveis de energia e resiliência mental enquanto se trabalha, vontade de investir no trabalho e persistência em situações de dificuldade. A dedicação refere-se a estar fortemente envolvido no trabalho, experienciando uma sensação de significância, entusiasmo, inspiração, orgulho e desafio. A absorção é caracterizada pela elevada concentração e envolvimento no trabalho, com a perceção pelo indivíduo da rápida passagem do tempo, tendo este dificuldade em abdicar do trabalho. ${ }^{8} \mathrm{~A}$ experiência e as emoções positivas do engagement no trabalho deverão facilitar comportamentos vantajosos, como o aumento da ligação à organização e o desempenho profissional. ${ }^{9}$ Para avaliar o grau de engagement do profissional com o trabalho foi aplicada a Utrecht Work Engagement Scale (UWES), validada para a língua portuguesa. ${ }^{2}$

A associação entre BEP e engagement no trabalho tem vindo a ganhar um reconhecimento crescente na literatura ao longo dos últimos anos. ${ }^{6-7} \mathrm{Tem}$ sido estudada a associação entre BEP e outcomes individuais positivos, como a moral e a saúde, o que por sua vez conduz a uma melhoria do desempenho organizacional, principalmente através do aumento da produtividade e da diminuição do absentismo laboral. O BEP e o engagement são, assim, duas entidades com forte correlação e que se reforçam mutuamente. ${ }^{10}$

Não foram encontrados estudos realizados em Portugal que avaliem o BEP nos profissionais de saúde ou a associação entre o BEP e o engagement no trabalho nesta população. Quanto ao engagement em profissionais de saúde, um estudo português refere que os enfermeiros de um ACeS apresentam um elevado nível de engagement ${ }^{11}$ e outro estudo, realizado em profissionais de saúde do ACeS de Cascais, concluiu que as USF modelo B possuíam maior engagement no trabalho, sendo este valor significativamente diferente do valor dos profissionais que trabalhavam em UCSP. ${ }^{12}$

$\mathrm{Na}$ literatura alguns autores conceptualizam o engagement como a antítese do burnout. ${ }^{8} \mathrm{Um}$ trabalho recente português sobre a prevalência do burnoutconcluiu que a ocorrência desta síndroma em profissionais de saúde portugueses é frequente, apesar de não se encontrar diferença estatisticamente significativa entre enfermeiros e médicos ou entre locais de trabalho. ${ }^{13}$
O objetivo geral deste estudo é testar a associação entre BEP e engagement no trabalho em profissionais dos cuidados de saúde primários portugueses, uma vez que não existem estudos neste âmbito. Como objetivo secundário pretende-se quantificar o BEP e o engagement de cada grupo profissional e unidade funcional (UF), bem como identificar as variáveis associadas ao BEP e engagement no trabalho.

\section{MÉTODOS}

Desenho do estudo

Realizou-se um estudo transversal analítico a partir de uma amostra de base institucional. Utilizou-se um questionário composto por duas partes para avaliar o BEP e o engagement dos profissionais de saúde. A primeira parte consistia num formulário construído pelos investigadores, tendo em conta a pesquisa bibliográfica efetuada, ${ }^{2,12,14-16}$ que incluiu questões sobre características sociodemográficas: local de trabalho, idade, sexo, estado civil (solteiro, casado/união de facto, viúvo, divorciado/separado), grupo profissional (assistente de medicina geral e familiar (MGF), interno de formação específica de MGF, enfermeiro, secretário clínico), presença de doença crónica, presença de filhos e número de anos de trabalho. A segunda parte apresentava dois questionários relativos às escalas OHQ para quantificação do BEP e UWES para avaliação do engagement no local de trabalho (outcome primário). O OHQ consiste numa escala unidimensional com 29 itens, em que os indivíduos responderam a cada um dos itens de acordo com uma escala de Likert que varia de um a seis (discordo sempre - concordo sempre). O score é posteriormente classificado em sete categorias, desde a classificação de «infeliz» até «demasiado feliz». A UWES é constituída por 17 itens, sendo seis de vigor, cinco de dedicação e seis de absorção. Nesse sentido, os participantes responderam a cada um dos itens de acordo com uma escala de Likert, cujos valores variam entre zero, se nunca tiveram esse sentimento ou crença, e seis, se o tiveram sempre. ${ }^{2}$

\section{Contexto e população do estudo}

A população do estudo englobou os profissionais de saúde das USF e UCSP dos Agrupamentos dos Centros de Saúde (ACeS) Grande Porto IV e Matosinhos. Incluíram-se todos os profissionais que integram estas uni- 
dades: médicos (assistentes de MGF e internos de formação específica de MGF), enfermeiros e secretários clínicos. Foram excluídos estudantes de medicina e de enfermagem, internos do ano comum, assistentes operacionais, telefonistas e elementos de serviços externos (empresas de limpeza e de segurança), visto se tratarem de profissionais que não integram de forma permanente as equipas de saúde.

\section{Aplicação dos questionários}

Convidaram-se os profissionais de saúde a participar no estudo, numa primeira fase por via eletrónica (e-mail institucional) e, dois meses depois, através de carta endereçada a cada unidade, de acordo com o parecer do Conselho Clínico de Saúde de cada ACeS. Nesta segunda fase solicitou-se aos profissionais que já tivessem respondido ao questionário por $e$-mail, que não $\mathrm{o}$ voltassem a preencher. Os questionários em formato papel foram recolhidos por UF, em envelopes fechados.

Solicitou-se o preenchimento do consentimento informado e assegurou-se o anonimato através da ausência de identificação direta dos inquiridos. A distribuição dos questionários foi efetuada entre novembro de 2015 e janeiro de 2016.

\section{Análise estatística}

Realizou-se a análise descritiva, apresentando frequências e percentagens para as variáveis categóricas, médias e desvios-padrão ou medianas para as variáveis contínuas e amplitude interquartil para as distribuições assimétricas.

Não se consideraram para análise de dados os questionários com missing values superiores a $20 \%$ por questionário. Utilizou-se o teste Alpha de Cronbach $(\alpha)$ para avaliar a consistência interna de cada escala, considerando-se um valor adequado se superior a 0,70 (valor mínimo proposto por Nunnally e Bernstein). ${ }^{17}$

Visando testar a associação das escalas UWES e OHQ entre si e das escalas com a idade e com o número de anos de trabalho utilizou-se o teste de correlação de Pearson. Para testar diferenças nas escalas entre as categorias das variáveis sexo, presença de doença crónica e presença de filhos usou-se o teste $t$ independente.

Utilizou-se o teste ANOVA para comparar as escalas entre os diferentes tipos de modelo de UF, estado civil e grupo profissional.
Usou-se um modelo de regressão linear múltipla para determinar a associação entre o BEP (OHQ) e o engagement (UWES), controlando para possíveis variáveis de confundimento, nomeadamente o sexo, a idade e o número de anos de trabalho. Considerou-se o OHQ como variável independente e o UWES como dependente.

Adotou-se um nível de significância de 0,05. Realizou-se a análise de dados utilizando o software Statistical Package for the Social Sciences (SPSS), versão 23.0®.

O protocolo do estudo foi aprovado pelos Conselhos Clínicos de Saúde dos ACeS Grande Porto IV e Matosinhos e pela Comissão de Ética da Administração Regional de Saúde do Norte.

\section{RESULTADOS}

Após aplicação dos critérios de exclusão, 598 profissionais de um total de 845 foram elegíveis para o estudo. Receberam-se 281 questionários, correspondentes a uma percentagem global de resposta de $47,0 \%$ (49,8\% para o ACeS Matosinhos e 50,2\% para o ACeS Grande Porto IV). Do total dos 281 questionários recebidos, $60,9 \%(n=171)$ foram entregues via $e$-mail e $39,1 \%$ $(n=110)$ via correio postal. Na análise de dados foi salvaguardada a duplicação de respostas, que não se verificou, sendo assim reduzida esta possibilidade.

Assumiram-se para a análise de dados 279 questionários. A percentagem de células com missing values foi de $0,61 \%$ (Figura 1 ).

Verificou-se uma maior participação dos assistentes de MGF (37,1\%), seguindo-se dos enfermeiros $(24,4 \%)$, secretários clínicos (22,5\%) e internos de MGF (16,0\%). Quando considerado o universo constituído pela totalidade de profissionais de ambos os ACeS, os assistentes de MGF foram também os mais participativos (55,7\%), seguidos dos internos de MGF (48,9\%), dos secretários clínicos $(44,9 \%)$ e dos enfermeiros (35,8\%).

A maioria dos participantes no estudo era do sexo feminino (81,6\%). A média de idade foi de $40,8 \pm 10,7$ anos e a média de anos de trabalho foi de 16,5 $\pm 11,2$ anos. Entre os profissionais, $62,8 \%$ eram casados ou estavam em união de facto, $65,2 \%$ tinham filhos e $71,4 \%$ trabalhavam em USF modelo B (Quadro I).

Para o UWES obteve-se um $\alpha_{\text {cronbach }}=0,93$ e para o $\mathrm{OHQ} \alpha_{\text {cronbach }}=0,86$, valores acima do recomendado e se- 


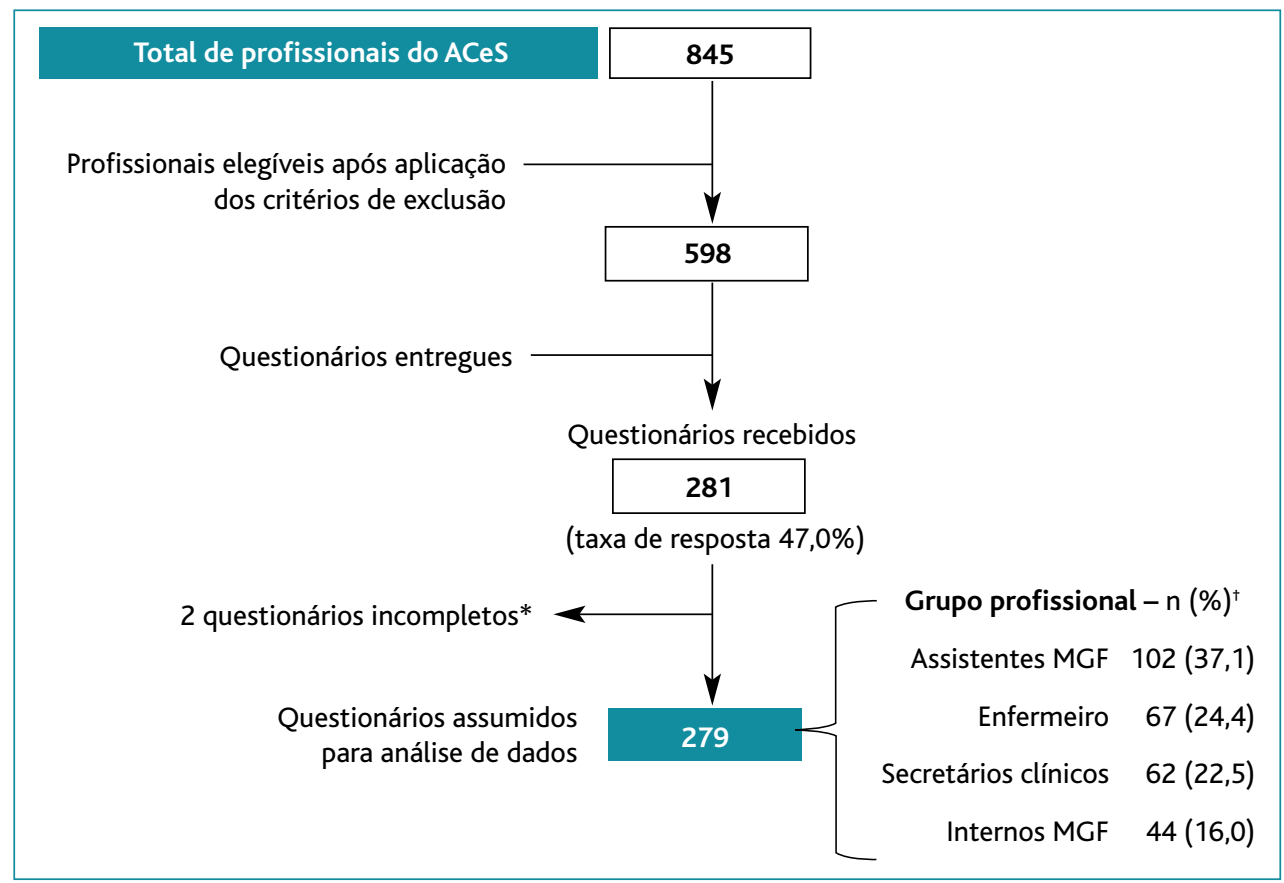

Figura 1. Fluxo de participantes ao estudo.

Abreviaturas: $\mathrm{ACeS}=$ Agrupamento de centros de saúde; MGF = Medicina geral e familiar .

* Questionários com missing values $>20 \%$; $+n=275$

\section{QUADRO I. Análise descritiva da população em estudo}

\begin{tabular}{l|c|r} 
Idade (anos) - média $\pm \mathrm{dp}$ & 40,8 & $\pm 10,7$ \\
\hline Sexo feminino $-n(\%)$ & 226 & $(81,6)$ \\
\hline Estado civil - $n$ (\%) & 174 & $(62,8)$ \\
$\quad$ Casado & 73 & $(26,4)$ \\
$\quad$ Solteiro & 27 & $(9,7)$ \\
$\quad$ Divorciado & 3 & $(1,1)$ \\
$\quad$ Viúvo & 180 & $(65,2)$ \\
\hline Filhos (sim)tm - $n(\%)$ & 216 & $(78,3)$ \\
\hline Doença crónica (não) $-n(\%)$ & 16,5 & $\pm 11,2$ \\
\hline Anos de trabalho - média $\pm d p$ & & \\
\hline Unidade Funcional - $n(\%)$ & 193 & $(71,4)$ \\
$\quad$ USF modelo B & 59 & $(21,9)$ \\
$\quad$ USF modelo A & 18 & $(6,7)$
\end{tabular}

Abreviaturas: USF = Unidade de saúde familiar; UCSP = Unidade de cuidados de saúde personalizados; $d p=$ desvio-padrão.

† Com filhos (com, pelo menos, um filho no agregado familiar).

* Ausência de doença crónica (e.g., diabetes mellitus, hipertensão arterial, insuficiência cardíaca, doença pulmonar obstrutiva crónica, lúpus, hepatite crónica ou outra elegível como doença crónica). melhante aos estudos de validação para a língua portuguesa (UWES $\alpha_{\text {cronbach }}=0,90^{2}$ e OHQ $\alpha_{\text {cronbach }}=0,89$ ). ${ }^{7}$

Verificou-se que $52,7 \%$ dos profissionais consideravam ser felizes ou bastante felizes ( $n=147$ ) e que $45,5 \%$ apresentavam um alto nível de engagement no trabalho ( $n=127)$, conforme descrito no Quadro II.

Confirmou-se a existência de uma correlação positiva entre as escalas OHQ e UWES $(r=0,32, p<0,05)$. Verificou-se uma correlação positiva entre o engagement, a idade e os anos de trabalho ( $r=0,16, p=0,008$ e $r=0,16$, $p=0,010)$ e uma correlação negativa entre o BEP, a idade e os anos de trabalho $(r=-0,14, p=0,019$ e $r=-0,13$, $p=0,034)$. Não se encontraram diferenças estatisticamente significativas no engagement no trabalho ou no BEP entre grupo profissional, tipo de UF, estado civil, existência de filhos e de doença crónica.

Ajustou-se um modelo de regressão linear múltipla para determinar a associação entre o OHQ e a UWES, controlando para as possíveis variáveis de confundimento, identificadas na análise exploratória, que incluíram a idade, o sexo e o número de anos de trabalho (Quadro III). Verificou-se uma associação significativa 


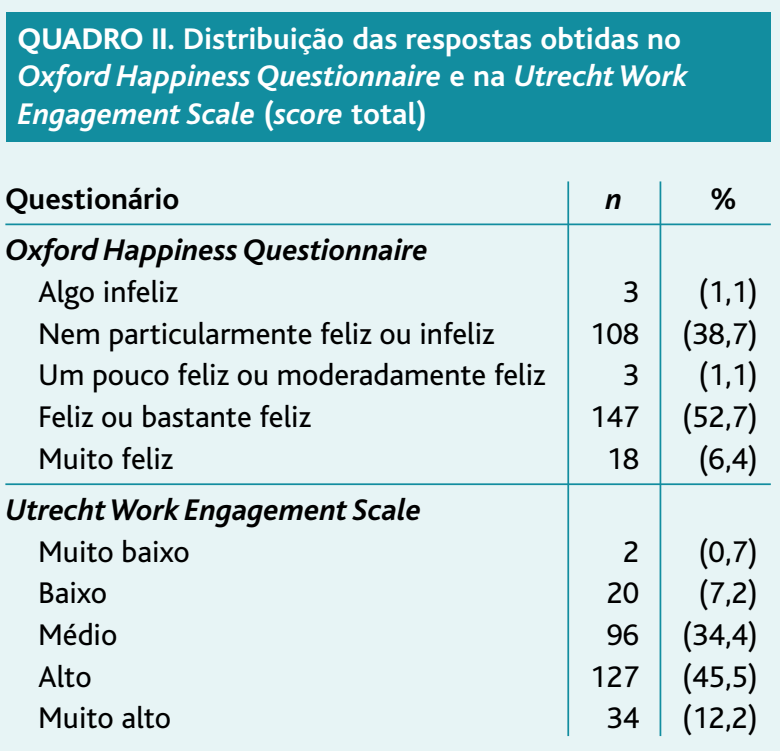

entre o engagement e o BEP $(\beta=0,621$, IC95\% $[0,423$ $0,818]$ ), controlando para a idade, sexo e número anos de trabalho.

\section{DISCUSSÃO}

Dado o contexto organizativo atual dos CSP em Portugal, que desde 2007 inclui unidades de prestação de cuidados de saúde com equipas multiprofissionais e com grau variável de autonomia organizacional e de incentivos, ${ }^{18}$ urge perceber qual o efetivo estado atual do engagement e da felicidade dos profissionais de saúde envolvidos.

A maioria dos profissionais que respondeu aos ques- tionários era feliz ou bastante feliz (52,5\%). Não foram encontrados quaisquer estudos que aplicassem o OHQ nos profissionais de saúde em Portugal.

Os profissionais que participaram no estudo apresentavam um alto nível de engagement no trabalho (45,5\%). Um estudo realizado no ACeS de Cascais verificou igualmente um nível elevado, superior nos profissionais das USF Modelo B, sendo que esta diferença era apenas estatisticamente significativa quando comparada com os profissionais das UCSP. ${ }^{12}$ No presente estudo não se encontraram diferenças estatisticamente significativas entre os diferentes modelos organizacionais.

Um dos achados relevantes deste trabalho foi a presença de uma correlação positiva e estatisticamente significativa entre o score do OHQ e da UWES, de acordo com o que é debatido na literatura. ${ }^{9-20} \mathrm{Se}$, por um lado, os profissionais mais felizes estão mais envolvidos na sua atividade laboral e são mais produtivos e eficazes, por outro, são estes mesmos profissionais, mais capacitados e satisfeitos no seu local de trabalho, que apresentam um maior grau de bem-estar ou felicidade. ${ }^{19-20}$

Neste estudo, a idade e o número de anos de trabalho estavam significativamente associados de forma negativa ao BEP. A literatura diz-nos que felicidade global aumenta dos 18 aos 45 anos, particularmente à custa do sentido de família e da felicidade com o emprego, diminuindo posteriormente, relacionando-se este facto com uma menor satisfação com o estado de saúde. ${ }^{14}$ Estes achados revestem-se de importância na me-

\begin{tabular}{|c|c|c|c|c|c|c|}
\hline & \multicolumn{6}{|c|}{ UWES } \\
\hline & \multicolumn{3}{|c|}{ Modelo não ajustado } & \multicolumn{3}{|c|}{ Modelo ajustado } \\
\hline & $\beta(95 \%$ IC) & Valor $p$ & Bstd & $\beta(95 \%$ IC) & Valor $p$ & Bstd \\
\hline Score OHQ & $0,570(0,372-0,767)$ & $p<0,000$ & 0,323 & $0,621(0,423-0,818)$ & $p<0,000$ & 0,354 \\
\hline Idade (anos) & - & - & - & $0,011(-0,019-0,041)$ & $p=0,473$ & 0,128 \\
\hline Sexo (feminino) & - & - & - & $-0,137(-0,405-0,132)$ & $p=0,317$ & $-0,057$ \\
\hline N. ${ }^{\circ}$ anos de trabalho & - & - & - & $0,007(-0,022-0,036)$ & $p=0,626$ & 0,087 \\
\hline
\end{tabular}

$R^{2}=0,15, R_{a}^{2}=0,14$.

Abreviaturas: $\mathrm{OHQ}=$ Oxford Happiness Questionnaire; UWES = Utrecht Work Engagement Scale; std = standardizado, $\mathrm{n}^{\circ}=$ número. 
dida em que não existe evidência publicada que aplique o mesmo instrumento em profissionais de saúde.

Quanto à aplicação da UWES, verificou-se que a idade e o número de anos de trabalho estão significativamente associados de forma positiva ao engagement. Estudos nos cuidados de saúde revelam que o engagement no trabalho aumenta com a idade e com o tempo de serviço, o que poderá traduzir a maior maturidade e experiência destes profissionais. ${ }^{2,15}$

Não se verificou existência de diferenças estatisticamente significativas entre sexo, estado civil, existência de filhos e doença crónica. Um estudo realizado nos CSP portugueses também não encontrou uma relação estatisticamente significativa entre engagement e sexo. ${ }^{2}$ No entanto, um estudo conduzido em enfermeiros portugueses demonstrou que os indivíduos casados ou em união de facto apresentavam maior engagement que os solteiros, viúvos e divorciados. ${ }^{15}$

Também não se encontraram diferenças estatisticamente significativas entre o engagement e os vários grupos profissionais, contrariamente ao verificado num estudo realizado nos CSP portugueses, no qual os secretários clínicos apresentavam maior engagement no trabalho, seguidos dos enfermeiros e, por fim, dos médicos. $^{2}$

Um ponto a destacar deste trabalho é a elevada taxa de participação dos profissionais selecionados. Obteve-se uma taxa global de resposta de $47,0 \%$, sendo que o ACeS Grande Porto IV foi responsável por 50,2\% das respostas e o ACeS de Matosinhos por 49,8\%.

A comparação entre os valores de $\beta$ não ajustado e ajustado para o sexo, a idade e o número de anos de trabalho é inferior a $15 \%$, pelo que se espera que o efeito das variáveis de confundimento não seja relevante na associação entre engagement no trabalho e BEP.

Este estudo apresenta algumas limitações, nomeadamente o potencial viés de desejabilidade social que poderá afetar a veracidade e validade dos dados, o facto de o conceito de felicidade ser subjetivo e variável para cada indivíduo, com difícil quantificação por questionários de todos os fatores associados, bem como o facto das respostas do inquirido poderem sofrer variabilidade dada a aplicação pontual dos instrumentos. Poderá igualmente ter ocorrido um viés no número de respostas obtidas, na medida em que o questionário foi reenviado por carta dois meses depois, sendo que não se verificou duplicação das respostas durante a análise de dados.

Este trabalho de investigação não descarta a possibilidade de o bem-estar no trabalho e a satisfação com a própria vida poderem ser mutuamente influenciáveis. Maior idade e, consequentemente, carreiras profissionais mais longas, foram as únicas variáveis encontradas que poderão ter impacto (positivo ou negativo), em ambas as dimensões estudadas, na vida de um trabalhador.

Os autores debruçaram-se sobre uma temática fundamental no contexto organizativo dos CSP, conferindo um incentivo para que se implementem estratégias que promovam o desenvolvimento pessoal e em equipa nas unidades de saúde, área que merece atenção por parte dos administradores do sistema, dos gestores das unidades, das ordens, associações e sindicatos.

Atendendo ao baixo valor de missing values e à proporção de participação elevada, os autores esperam um limitado viés de seleção, com um diminuto impacto nos resultados apresentados. Deste modo, garante-se a possibilidade da inferência dos resultados para a população estudada.

Seria importante a realização de outros estudos longitudinais para identificar a causalidade entre as variáveis avaliadas neste trabalho, bem como avaliar a relação de outros fatores, como o burnout, a produtividade e o envolvimento organizativo. Seria também útil a realização de estudos semelhantes noutros ACeS, atendendo a que existem poucos trabalhos nesta área que envolvam todos os grupos profissionais que trabalham nos CSP.

\section{REFERÊNCIAS BIBLIOGRÁFICAS}

1. Gavin JH, Mason RO. The virtuous organization: the value of happiness in the workplace. Organ Dyn. 2004;33(4):379-92.

2. Martins JN. Validação da versão portuguesa do questionário Utrecht Work Engagement para medir o engagement no trabalho dos profissionais dos cuidados de saúde primários [Dissertation]. Lisboa: ISCTE; 2013.

3. Sauter SL, Lim SY, Murphy LR. Organizational health: a new paradigm for occupational stress research at NIOSH. J Occup Ment Health. 1996;4(4):248-54.

4. Ozcakir A, Dogan FO, Cakir YT, Bayram N, Bilgel N. Subjective wellbeing among primary health care patients. PLoS One. 2014;9(12): e114496.

5. Wright TA, Cropanzano R. The role of psychological well-being in job performance: a fresh look at an age-old quest. Organ Dyn. 2004;33(4): 338-51.

6. Hills P, Argyle M. The Oxford Happiness Questionnaire: a compact scale 
for the measurement of psychological well-being. Pers Individ Dif. 2002;33(7):1073-82.

7. Schaufeli WB, Salanova M, González-Romá V, Bakker AB. The measurement of engagement and Burnout: a two sample confirmatory factor analytic approach. J Happiness Stud. 2002;3(1):71-92.

8. Bakker AB, Schaufeli WB, Leiter MP, Taris TW. Work engagement: an emerging concept in occupational health psychology. Work Stress. 2008;22(3):187-200.

9. Alarcon GM. Edwardes, JM. The relationship of engagement, job satisfaction and turnover intentions. Stress Health. 2011;27(3):e294-8.

10. Robertson IT, Birch AJ, Cooper CL. Job and work attitudes, engagement and employee performance: where does psychological well-being fit in? Leadersh Organ Dev J. 2012;33(3):224-32.

11. Correia VM. Engagement e suporte social nos enfermeiros de cuidados de saúde primários [Dissertation]. Porto: Escola Superior de Enfermagem do Porto; 2012.

12. Garcia SA. Engagement no trabalho dos profissionais das Unidades de Cuidados de Saúde Primários e a sua relação com a performance e a satisfação no trabalho [Dissertation]. Lisboa: ISCTE; 2012.

13. Maroco J, Tecedeiro M, Martins P, Meireles A. O Burnout como factor hierárquico de $2^{\mathrm{a}}$ ordem da Escala de Burnout de Maslach. Anal Psicol. 2008;26(4):639-49.

14. Easterlin RA. Life cycle happiness and its sources: intersections of psychology, economics, and demography. J Econ Psychol. 2006;27(4) 463-82.

15. Dias S, Queirós C. Burnout e engagement em enfermeiros portugue- ses. In: UNIESEP, editor. Saúde e qualidade de vida: uma meta a atingir. Porto: Escola Superior de Enfermagem do Porto; 2011. p. 300-8. ISBN 9789899610354

16. Silva M, Queirós C, Cameira M, Vara N, Galvão A. Burnout and engagement among health professionals from interior-north of Portugal [Burnout and engagement among health professionals from interiornorth of Portugal]. Psicol Saúde Doenç. 2015;16(3):286-99. Portuguese

17. Nunnally JC, Bernstein IH. Psychometric theory. 3rd ed. New York: McGraw-Hill; 1994. ISBN 9780070478497

18. Decreto-Lei n. ${ }^{\circ}$ 298/2007, de 22 de agosto de 2007. Diário da República. $1^{\text {a }}$ Série(161).

19. Rego A. Empregados felizes são mais produtivos? [Internet]. Aveiro: Universidade de Aveiro; 2006. Available from: https://www.ua.pt/egi/ ReadObject.aspx?obj=7978

20. Zanelli JC, Borges-Andrade JE, Bastos AV. Psicologia, Organizações e Trabalho no Brasil. Porto Alegre: Artmed; 2005. ISBN 9788536303642

\section{CONFLITO DE INTERESSES}

Os autores declaram não ter quaisquer conflitos de interesse.

\section{ENDEREÇO PARA CORRESPONDÊNCIA}

Sara Cunha

E-mail: samicunha@gmail.com

Recebido em 30-03-2017

Aceite para publicação em 15-01-2018

\section{ABSTRACT}

\section{WORK HAPPINESS AND ENGAGEMENT IN PRIMARY HEALTH CARE}

Objectives: To test the association between psychological well-being (PWB) and health-care professionals' work engagement. Type of Study: Cross-sectional analytical study.

Setting: Matosinhos Group of Health Centers and Grande Porto IV Group of Health Centers.

Population: Healthcare professionals from the two Groups of Health Centers.

Methods: We used the Oxford Happiness Questionnaire (OHQ) and the Utrecht Work Engagement Scale (UWES), validated for the Portuguese language, to evaluate the PWB and work engagement, respectively. Using a multiple linear regression model, we tested the relation between PWB and work engagement, controlling for several confounding variables.

Results: Between the professionals, $52.5 \%$ are happy or quite happy and $45.5 \%$ show a high engagement level. There is a significant correlation between both scales $(r=0.32, p<0.05)$. This correlation is maintained even when controlled for confounding variables $(\beta=0.621, \mathrm{Cl} 95 \%$ [0.423-0.818]). Age and years of work are correlated with the UWES $(r=0.16, p=0.008$ and $r=0.16$, $p=0.010)$ and with the OHQ $(r=-0.14, p=0.02$ and $r=-0.13, p=0.03)$.

Conclusions: The correlation found between PWB and engagement was expected by the current literature. Higher age and service time correlate with engagement, as seen in previous studies, but to lower PWB. This study approaches an important theme in the Primary Health Care field, suggesting the implementation of strategies that promote personal and team development.

Keywords: Work; Happiness; Primary health care; Job satisfaction; Occupational health. 